\title{
DRAMATIC SPECTRAL EVOLUTION OF WZ Sge DURING THE 2001 SUPEROUTBURST
}

\author{
D. Nogami ${ }^{1}$ and T. Iijima ${ }^{2}$
}

We spectroscopically observed WZ SGE in 11 nights during the 2001 superoutburst. The feature of the spectra dramatically varied from at the outburst maximum through during the rebrightening phase.

WZ SGE is the prototypical star of WZ SGE-type st,ars, a small class of SU UMa-type dwarf novae. WZ SGE stars have unusual characteristics of quite large outburst amplitudes up to $\sim 8 \mathrm{mag}$, extraordinary long recurrence cycles of the outbursts ( $\geq 10$ years), no (or only a few) normal outbursts.

A new outburst of WZ SGE was caught on 2001 July 23, when 23 years had passed since the last outburst (see e.g. Patterson et al. 2002). This outburst consisted of a main outburst for $\sim 25$ days, a dip for $\sim 4$ days, a rebrightening phase including 12 maxima for $\sim 20$ days, and a fading tail lasting over 10 days. We took optical spectra of WZ SGE during this outburst, covering the period from the outburst maximum through the ninth maximum in the rebrightening phase.

Figure 1 shows the spectra obtained at the first night, which has a very blue continuum with a variety of emission/absorption lines. Emergence of the high-excitation emission lines of $\mathrm{He}, \mathrm{C}$, and $\mathrm{N}$, and the evolution from absorption to emission of $\mathrm{H} \alpha$ is a new evidence of the outside-in type outburst due to the disk instability. These emission lines are supposed to originate from the chromosphere of the accretion disk formed by irradiation.

The peak separations of $\mathrm{H} \alpha$ and $\mathrm{He}$ II 4686 were about $700 \mathrm{~km} \mathrm{~s}^{-1}$, which is a half of that of $\mathrm{H} \alpha$ in quiescence, at the very early phase of the outburst. This implies that the accretion disk extended, perhaps to have a circumstellar part.

$\mathrm{Na}$ I $\mathrm{D}$ was found to be in strong absorption during the main outburst. This stationary line indicates that a cool region might exist around the center of mass of this system, or the origin of this line may be the circumstellar part of the disk.

During the rebrightening phase, the spectra

\footnotetext{
${ }^{1}$ Hida Observatory, Kyoto University, Kamitakara, Gifu 506-1314, Japan (nogami@kwasan.kyoto-u.ac.jp).

${ }^{2}$ Astronomical Observatory of Padova, Asiago Section, Osservatorio Astrofisico, I-36012 Asiago (Vi), Italy (iijima@ astras.pd.astro.it).
}
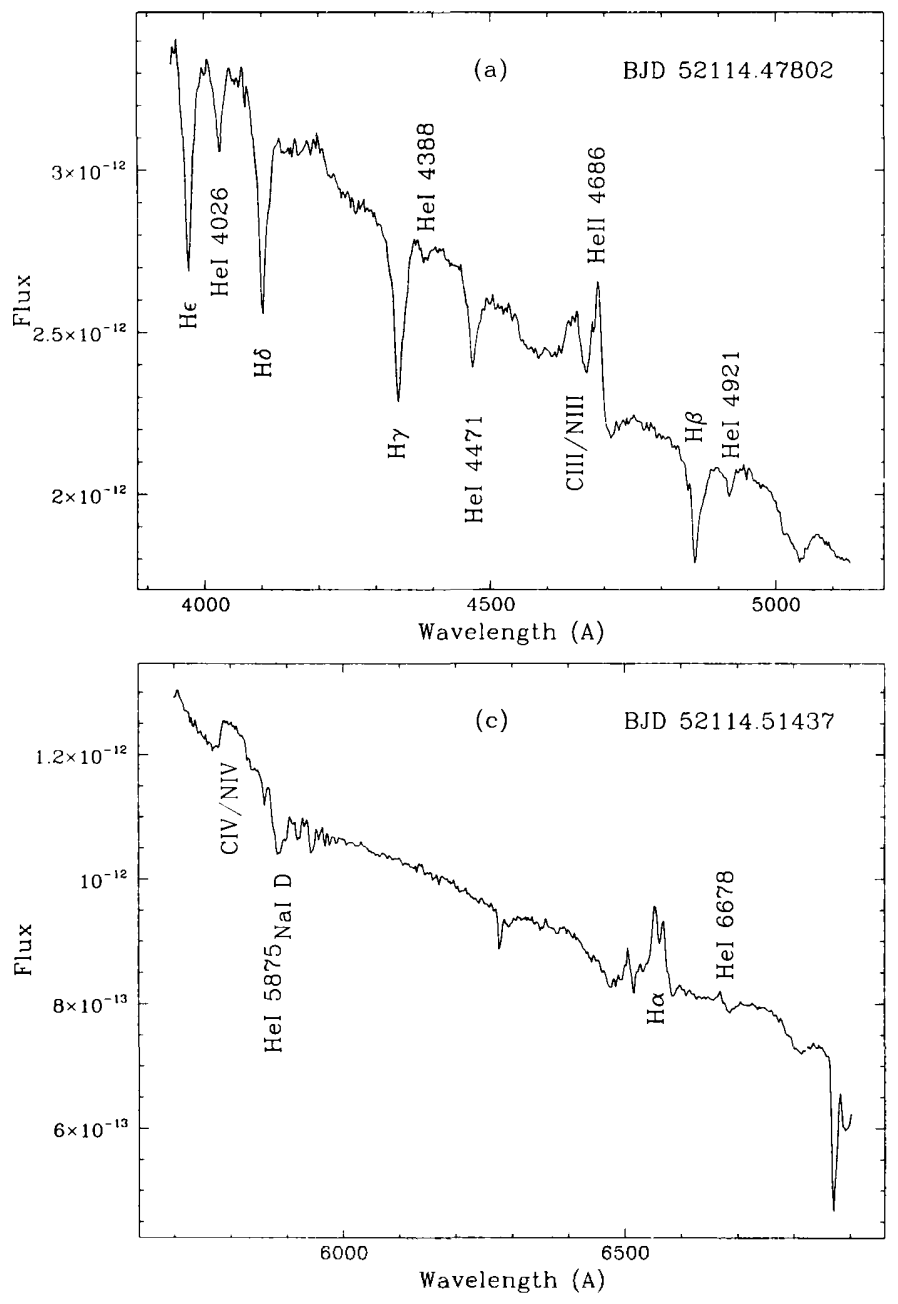

Fig. 1. Spectra around the outburst maximum. We can see emission lines of $\mathrm{H} \alpha, \mathrm{He}$ II $4686, \mathrm{C}$ III/N III around 4630 , and C IV/N IV around 5800, and absorption lines of other Balmer lines, $\mathrm{He}$, and $\mathrm{Na}$ I D on a blue continuum.

showed absorption lines of $\mathrm{H}$ and $\mathrm{He} \mathrm{I}$ at the maximum and emission lines of the same species at the bottom, which means that the state transition of the disk was due to propagation of the heating/cooling waves, as in normal outbursts of usual dwarf novae.

The details of these observations and more discussion will be published by Nogami \& Iijima (2004).

\section{REFERENCES}

Patterson, J., et al. 2002, PASP, 114, 721

Nogami, D., \& lijima, T. 2004, PASJ, in press 